\title{
Joint Scalar versus Joint Velocity-Scalar PDF Simulations of Bluff-Body Stabilized Flames with REDIM
}

\author{
B. Merci • B. Naud · D. Roekaerts • U. Maas
}

Received: 13 December 2007 / Accepted: 22 May 2008 / Published online: 3 July 2008

(C) Springer Science + Business Media B.V. 2008

\begin{abstract}
Two transported PDF strategies, joint velocity-scalar PDF (JVSPDF) and joint scalar PDF (JSPDF), are investigated for bluff-body stabilized jet-type turbulent diffusion flames with a variable degree of turbulence-chemistry interaction. Chemistry is modeled by means of the novel reaction-diffusion manifold (REDIM) technique. A detailed chemistry mechanism is reduced, including diffusion effects, with $\mathrm{N}_{2}$ and $\mathrm{CO}_{2}$ mass fractions as reduced coordinates. The second-moment closure RANS turbulence model and the modified Curl's micro-mixing model are not varied. Radiative heat loss effects are ignored. The results for mean velocity and velocity fluctuations in physical space are very similar for both PDF methods. They agree well with experimental data up to the neck zone. Each of the two PDF approaches implies a different closure for the velocity-scalar correlation. This leads to differences in the radial profiles in physical space of mean scalars and mixture fraction variance, due to different scalar flux modeling. Differences are visible in mean mixture fraction and
\end{abstract}

B. Merci

Postdoctoral Fellow of the Fund of Scientific Research, FWO-Vlaanderen, Flanders, Belgium

B. Merci $(\bowtie)$

UGent, Department of Flow, Heat and Combustion Mechanics, Ghent University, Sint-Pietersnieuwstraat 41, 9000 Ghent, Belgium

e-mail: Bart.Merci@UGent.be

B. Naud

Energy Department, Modeling and Numerical Simulation Group, Ciemat, Madrid, Spain

D. Roekaerts

Department of Multi-Scale Physics, Delft University of Technology, Delft, The Netherlands

U. Maas

Institute for Technical Thermodynamics, Karlsruhe University (TH), Germany 
mean temperature, as well as in mixture fraction variance. In principle, the JVSPDF simulations can be closer to physical reality, as a differential model is implied for the scalar fluxes, whereas the gradient diffusion hypothesis is implied in JSPDF simulations. Yet, in JSPDF simulations, turbulent diffusion can be tuned by means of the turbulent Schmidt number. In the neck zone, where the turbulent flow field results deteriorate, the joint scalar PDF results are in somewhat better agreement with experimental data, for the test cases considered. In composition space, where results are reported as scatter plots, differences between the two PDF strategies are small in the calculations at hand, with a little more local extinction in the joint scalar PDF results.

Keywords CFD - Transported PDF $\cdot$ REDIM $\cdot$ Bluff body stablised flames

\section{Introduction}

In turbulent non-premixed flames, non-linear interaction between turbulence and finite-rate chemistry is often important. Turbulence-chemistry interaction is thus a central issue in non-premixed turbulent flame modeling and simulations. Strong interaction may lead to local extinction or incomplete combustion. Under such circumstances, pre-assumed PDF modeling is typically not suitable. Given a certain chemistry model, an exact treatment of the chemical reaction source term is achieved in the framework of the computationally much more expensive transported probability density function (PDF) technique, be it joint scalar or joint velocity-scalar [1]. The conditional molecular flux is left as a modeling issue.

In this study, we apply transported PDF methods, based on stochastic Lagrangian modeling. We compare the results of joint scalar PDF (JSPDF) and joint velocityscalar PDF (JVSPDF) simulations. Following [1], a mass density function (MDF) transport equation is modeled and solved using a particle stochastic method. The three major modeling ingredients are: the turbulence model, the chemistry model and the micro-mixing model. Several recent comparative studies focus on the influence of these different sub-models on numerical simulation results of turbulent nonpremixed flames. In [2] e.g., three widely used micro-mixing models are compared, considering stochastic simulations of partially stirred reactors (PaSR). In the context of transported scalar PDF modeling, the same micro-mixing models are compared in [3] for the piloted jet diffusion flame Delft flame III, and in [4] for the bluffbody stabilized flames 'Sydney HM1-3' [5-7]. In [8], seven combustion mechanisms for methane/air are compared for joint velocity-scalar-turbulence frequency PDF calculations of the non-premixed piloted jet flames 'Sandia D-F'. In [9], the influence of three $C_{1}$ chemistry models on two micro-mixing models is studied for Delft flame III and the bluff-body flames HM1-3. In [10], detailed models, containing $C_{2}$ chemistry, are applied to the same bluff-body test cases. In [11], an algebraic extension of the modified Curl's coalescence/dispersion (CD) mixing model [12] is applied to HM2 and HM3, with systematically reduced $C_{2}$ chemistry.

In the present paper, we focus on the Sydney flames HM1 and HM3. The fuel is a mixture of $50 \% \mathrm{H}_{2} / 50 \% \mathrm{CH}_{4}$ by volume. It is a target test case of the TNF 
workshop series on turbulent non-premixed flames [13]. Recently, a few papers on these flames appeared, employing transported PDF $[10,11]$ and conditional moment closure (CMC) [14]. With respect to chemistry modeling, the present work is an improvement over [4]. Instead of a $C_{1}$ skeletal mechanism, the novel reactiondiffusion manifold (REDIM) technique [15] is applied, reducing a detailed reaction mechanism, consisting of 34 species and 302 reactions [16]. Recently, there has been much research activity in the field of reduced chemistry, in combination with chemistry-transport coupling. A comprehensive review is provided by Ren and Pope $[18,19]$, which argues in favor of the 'close-parallel' assumption for chemistry-based slow manifolds. This basically reduces to the assumption that the system evolves on a manifold, close and parallel to a manifold that is solely based on chemistry. The distance from the 'chemical' manifold is governed by diffusion processes. We apply the REDIM technique of [15], leading to an invariant low-dimensional manifold, directly accounting for the coupling of chemistry and diffusion (and convection). The obtained, invariant, manifold need not be parallel to a manifold that is solely based on chemistry.

In order to achieve good agreement to experimental data for the turbulent flow field, in terms of radial profiles in physical space of mean velocities and velocity fluctuations, a second moment closure model is applied with modified model constants $[4,20]$. Good quality of the results is obtained up to the neck zone. The modified Curl's coalescence/dispersion (CD) [21] is used as micro-mixing model. In [4], a comparative study is presented to the Euclidean Minimum Spanning Tree (EMST) model [22], in the framework of transported scalar PDF, revealing that EMST is not superior to CD for the test cases considered. We confirmed this with the current chemistry approach or REDIM (not discussed here). In [4], we reported it was not possible to obtain a stationary solution for HM3 with the CD mixing model. This was attributed to the use of $C_{1}$ chemistry in [4]. Applying reduced chemistry, with the REDIM technique, a stationary solution is obtained and profiles of major and minor chemical species in physical space are in good agreement to experimental data. We remark that Gkagkas et al. [11] report that, in line with our previous findings [4], no statistically stationary solution could be found for HM3 with their C/D type micromixing model, starting from a steady laminar flamelet solution.

The choice of the PDF description itself has direct consequences on the modeling of the scalar flux and higher order velocity-scalar correlations. With the joint scalar MDF, the gradient diffusion assumption for closure of the conditional fluctuating velocity term in the MDF transport equation, leads to a simple algebraic model for the scalar flux. In this case, good agreement in physical space for mean mixture fraction and mixture fraction variance can be obtained by tuning the turbulent Schmidt number. When the velocity components are included in the PDF description, the transport equation for the joint velocity-scalar MDF is modeled and solved using a particle method. In this case, the combination of the model for particle velocity evolution and the micro-mixing model implies a modeled transport equation for the scalar flux and higher order velocity-scalar correlations. In the present study, we compare results of JSPDF and JVSPDF simulations, using hybrid finite volume/particle methods, implemented in the same in-house computer program 'PDFD', described by Naud et al. in [23]. The same turbulence, chemistry and micro-mixing models are used in all simulations. 


\section{PDF Approach}

\subsection{Statistical description}

The statistical description of the flow is in terms of the joint one-point $\operatorname{PDF} f_{\phi}$ : $f_{\phi}(\boldsymbol{\psi} ; \boldsymbol{x}, t) . \mathrm{d} \boldsymbol{\psi}$ is the probability that $\boldsymbol{\Phi}$ is in the interval $[\boldsymbol{\psi}, \boldsymbol{\psi}+\mathrm{d} \boldsymbol{\psi}$ [at point $(\boldsymbol{x}, t)$. When JSPDF is considered, $\boldsymbol{\Phi}$ is the composition vector $\boldsymbol{\phi}$. For JVSPDF, $\boldsymbol{\Phi}=(\boldsymbol{U}, \boldsymbol{\phi})$, with $\boldsymbol{U}$ the velocity vector. The joint PDF is defined as in [1, 4]:

$$
f_{\phi}(\boldsymbol{\psi} ; \boldsymbol{x}, t)=\langle\delta[\boldsymbol{\phi}(\boldsymbol{x}, t)-\boldsymbol{\psi}]\rangle
$$

where $\delta[]$ is the Dirac delta function and where the brackets \langle\rangle refer to expected values [1]. Using the conditional expected value $\langle Q(\mathrm{x}, t) \mid \boldsymbol{\psi}\rangle f_{\phi}(\boldsymbol{\psi} ; \mathrm{x}, t)=$ $\langle Q(\mathrm{x}, t) . \delta[\boldsymbol{\phi}(\mathrm{x}, t)-\boldsymbol{\psi}])$, mean (or 'expected') values are defined as:

$$
\langle Q(\boldsymbol{x}, t)\rangle=\int_{[\psi]}\langle Q(\boldsymbol{x}, t) \mid \psi\rangle f_{\phi}(\boldsymbol{\psi} ; \boldsymbol{x}, t) d \boldsymbol{\psi}
$$

Fluctuations are defined as: $q^{\prime}(\boldsymbol{x}, t)=Q(\boldsymbol{x}, t)-\langle Q(\boldsymbol{x}, t)\rangle$.

For variable density flows, it is useful to consider the joint $\operatorname{MDF} F_{\phi}(\boldsymbol{\psi})=$ $\rho(\boldsymbol{\psi}) f_{\phi}(\boldsymbol{\psi})$. Density weighted averages (Favre averages) are:

$$
\widetilde{Q}(\mathrm{x}, t)=\frac{\langle\rho(\mathrm{x}, t) Q(\mathrm{x}, t)\rangle}{\langle\rho(\mathrm{x}, t)\rangle}=\frac{\int_{[\psi]}\langle Q(\mathrm{x}, t) \mid \boldsymbol{\psi}\rangle F_{\Phi}(\boldsymbol{\psi} ; x, t) d \boldsymbol{\psi}}{\int_{[\psi]} F_{\Phi}(\boldsymbol{\psi} ; x, t) d \boldsymbol{\psi}} .
$$

Fluctuations with respect to the Favre average are defined as: $q^{\prime \prime}(\boldsymbol{x}, t)=$ $Q(\boldsymbol{x}, t)-\widetilde{Q}(\boldsymbol{x}, t)$.

\subsection{Joint scalar PDF transport equation}

When the joints scalar MDF is considered, the following transport equation is modeled and solved [1]:

$$
\frac{\partial F_{\phi}}{\partial t}+\frac{\partial \widetilde{U}_{j} F_{\phi}}{\partial x_{j}}+\frac{\partial}{\partial \psi_{\alpha}}\left[S_{\alpha}(\boldsymbol{\psi}) F_{\phi}\right]=-\frac{\partial}{\partial x_{i}}\left[\left\langle u_{i}^{\prime \prime} \mid \boldsymbol{\psi}\right\rangle F_{\phi}\right]-\frac{\partial}{\partial \psi_{\alpha}}\left[\left\langle\theta_{\alpha} \mid \boldsymbol{\psi}\right\rangle F_{\phi}\right]
$$

with the mixing term $\theta_{\alpha}(\boldsymbol{\phi})=-\frac{1}{\rho(\boldsymbol{\phi})} \frac{\partial J_{j}^{\alpha}}{\partial x_{j}}$, and where $S_{\alpha}$ is the reaction source term for scalar $\phi_{\alpha}$ and $\boldsymbol{J}^{\alpha}$ its molecular flux. The first term on the right hand side is modeled using a gradient diffusion assumption:

$$
\frac{\partial}{\partial x_{i}}\left[\left\langle u_{i}^{\prime \prime} \mid \psi\right\rangle F_{\phi}\right]=-\frac{\partial}{\partial \mathrm{x}_{\mathrm{i}}}\left[\Gamma_{T} \frac{\partial\left(F_{\phi} / \rho\right)}{\partial x_{i}}\right]
$$

where $\Gamma_{T}$ is the turbulent diffusivity, modeled as $\Gamma_{T}=\mu_{T} / S c_{T}$, with $\mu_{T}$ the eddy viscosity. The turbulent Schmidt number is taken as $\mathrm{Sc}_{T}=0.7$ or $\mathrm{Sc}_{T}=0.85$ (see below). 


\subsection{Joint velocity-scalar PDF transport equation}

When velocity is included in the PDF description, the transport equation for the joint velocity-scalar MDF can be written (neglecting the mean viscous stress tensor gradient $\left.\frac{\partial\left\langle\tau_{i j}\right\rangle}{\partial x_{j}}\right)$ :

$$
\begin{aligned}
\frac{\partial F_{U \phi}}{\partial t} & +V_{j} \frac{\partial F_{U \phi}}{\partial x_{j}}+\left(-\frac{1}{\langle\rho\rangle} \frac{\partial\langle p\rangle}{\partial x_{j}}+g_{j}\right) \frac{\partial F_{U \phi}}{\partial x_{j}}+\frac{\partial}{\partial \psi_{\alpha}}\left[S_{\alpha}(\psi) F_{U \phi}\right] \\
& =-\frac{\partial}{\partial V_{j}}\left[a_{j} F_{U \phi}\right]-\frac{\partial}{\partial \psi_{\alpha}}\left[\frac{1}{\rho(\psi)}\left\langle-\frac{\partial J_{j}^{\alpha}}{\partial x_{j}} \mid V, \psi\right\rangle F_{U \phi}\right]
\end{aligned}
$$

The term $a_{j}$ denotes:

$$
a_{j}=\left(\frac{1}{\langle\rho\rangle}-\frac{1}{\rho(\psi)}\right) \frac{\partial\langle p\rangle}{\partial x_{j}}+\frac{1}{\rho(\psi)}\left\langle-\frac{\partial p^{\prime}}{\partial x_{j}}+\frac{\partial \tau_{i j}^{\prime}}{\partial x_{i}} \mid V, \psi\right\rangle \text {. }
$$

A Langevin model is used to close this term, as mentioned below, in the section on turbulence modeling. The terms on the left hand side of (6) are in closed form. Compared to (4), effects of convection and mean pressure gradient are now exactly taken into account.

\subsection{Hybrid finite volume/particle method}

Equations (4) and (6) are solved using the consistent hybrid finite-volume/particle method presented in [23]. Mean velocity $\widetilde{\boldsymbol{U}}$, mean pressure gradient $\partial\langle p\rangle / \partial x_{i}$, Reynolds stresses $\left\langle p u_{i}^{\prime \prime} u_{j}^{\prime \prime}\right\rangle$ and turbulent dissipation rate $\varepsilon$, which is not included in the PDF representation, are obtained by a standard finite-volume (FV) method based on a pressure-correction algorithm.

A particle method is applied for the solution of the MDF transport equation. A set of uniformly distributed computational particles evolves according to stochastic differential equations. Each particle has a set of properties $\left\{w^{*}, m^{*}, \boldsymbol{X}^{*}, \boldsymbol{\phi}^{*}\right\}$ (scalar MDF) or $\left\{w^{*}, m^{*}, \boldsymbol{X}^{*}, \boldsymbol{u}^{*}, \boldsymbol{\phi}^{*}\right\}$ (velocity-scalar MDF), where $w^{*}$ is a numerical weight, $m^{*}$ is the particle mass, $\boldsymbol{X}^{*}$ its position, $\boldsymbol{u}^{*}$ its fluctuating velocity and $\boldsymbol{\phi}^{*}$ the particle's composition. The superscript * denotes that the quantity is a particle property. Particle mass $m^{*}$ is constant in time. The particle joint scalar MDF is:

$$
F_{\phi}^{P}(\boldsymbol{x}, \boldsymbol{\psi} ; t)=\left\langle\sum_{*} w^{*} m^{*} \cdot \delta\left(\boldsymbol{X}^{*}(t)-\boldsymbol{x}\right) \cdot \delta\left(\boldsymbol{\phi}^{*}(t)-\boldsymbol{\psi}\right)\right\rangle
$$

Additional properties can be deduced for each stochastic particle from the primary properties listed above. As an example, the particle density is obtained as: $\rho^{*}(t)=$ $\rho\left[\boldsymbol{\phi}^{*}(t)\right]$. 
Increments of particle position $\boldsymbol{X}^{*}$ and composition $\boldsymbol{\phi}^{*}$ over a small time step $d t$ are given by:

$$
\begin{aligned}
& d X_{i}^{*}=\left(U_{i}^{*}+\left[U_{i}^{c}\right]^{*}\right) d t \\
& d \phi_{\alpha}^{*}=\theta_{\alpha}^{*} d t+S_{\alpha}\left(\phi^{*}\right) d t
\end{aligned}
$$

The correction velocity $\boldsymbol{U}^{c}$ results from a position correction algorithm [24], ensuring that the volume represented by the particles in a computational cell, equals the cell geometric volume. The values of the position correction algorithm parameters are set to: $k_{f}=0.5, k_{b}=1$ and $N_{T A}^{c}=100$, as in [23]. $\theta_{\alpha}^{*}$ represents the model for the conditional scalar flux (micro-mixing model).

With the scalar MDF, $\boldsymbol{U}^{*}$ results from a random walk model for particle position evolution:

$$
U_{i}^{*} d t=\left[\tilde{U}_{i}+\frac{1}{\langle\rho\rangle} \frac{\partial \Gamma_{T}}{\partial x_{i}}\right]^{*} d t+\left[\left(\frac{2 \Gamma_{T}}{\langle\rho\rangle}\right)^{1 / 2}\right]^{*} d W_{i}
$$

with $d W_{i}$ an increment over $d t$ of the Wiener process $W_{i}$.

With the joint velocity-scalar MDF, the equations read:

$$
\begin{gathered}
U_{i}^{*}=\left[\widetilde{U}_{i}\right]^{*}+u_{i}^{*} \\
d u_{i}^{*}=-u_{j}^{*}\left[\frac{\partial \widetilde{U}_{i}}{\partial x_{j}}\right]^{*} d t+\left[\frac{1}{\langle\rho\rangle} \frac{\partial\langle\rho\rangle \widetilde{u_{i}^{\prime \prime} u_{j}^{\prime \prime}}}{\partial x_{j}}\right]^{*} d t+a_{i}^{*} d t
\end{gathered}
$$

In the above equations, the quantities between brackets []* are FV properties interpolated at the particle location using bilinear basis functions as in [25]. The model for $a_{i}^{*}$ is specified below in the section on turbulence modeling.

The method of fractional steps [1] is used to integrate the system of equations. In order to ensure second-order accuracy, the 'midpoint rule' is used [24, 25]. A local time-stepping algorithm, developed in the framework of statistically stationary problems $[3,26]$, is applied.

\subsection{Consistency and coupling}

The turbulent dissipation rate is not included in the PDF representation. The transport equation solved for $\varepsilon$ in the FV method, provides additional information, required to model the unclosed term $a_{i}$ and the micro-mixing model. The other FV equations are consistent with the modeled MDF transport equation [23]. The mean density $\langle\rho\rangle$ in the FV method is directly obtained from the iteration averaged mean density in the particle method, applying the iteration averaging procedure as presented in [23]. We remark that the global convergence of the method is improved in comparison to the latter reference, where density relaxation was used.

An outer iteration consists of a number of FV iterations and particle time steps. We use a fixed number of particle time steps (typically five), while the FV method is iterated until the residuals of all equations are decreasing and the global mean 
pressure correction is below a specified threshold (with a maximum of $1000 \mathrm{FV}$ iterations per outer iteration).

\section{Modeling}

\subsection{Turbulence model}

Turbulence is modeled using the second-moment closure RANS model of [20]. This is the isotropization of production model of [27], with modified constant value $C_{\varepsilon 1}=1.6$, instead of the standard value $C_{\varepsilon 1}=1.44$. Consistently, the Lagrangian isotropization of production model (LIPM) is used in the velocity-scalar PDF approach to describe velocity evolution $a_{i}[28,29]$.

\subsection{Reduced chemistry model: REDIM}

The REDIM concept, described in detail in [15], is used to reduce a detailed $\mathrm{CH}_{4} / \mathrm{H}_{2}$ /air detailed reaction mechanism of 34 species and 302 reactions [16]. This concept is based on a relaxation process, where an initial guess for a low-dimensional manifold evolves in such a manner that an invariant slow reaction/diff usion manifold is obtained. One major advantage of the REDIM technique over the ILDM concept is the fact that a REDIM exists in the whole accessed domain, even at low temperatures (close to the unburned mixture), where chemistry is slow and ILDM does not yield an existing manifold. Close to equilibrium the REDIM is typically close to the ILDM. It has been shown in [15] that, if chemistry governs the overall process, i.e., gradients tend to zero, the REDIM concept yields slow manifolds or, equivalently, iteratively refined ILDMs as a limiting case [17].

The $m$-dimensional vector of reduced coordinates represents the composition vector $\boldsymbol{\phi}$. The evolution equation, solved to obtain the low-dimensional manifolds (see below), is formulated in generalized coordinates. In order to simplify the subsequent use of the tables, suitable simple progress variables are identified after the calculation of the REDIM. They are used as the reduced coordinates. For the test case under study, it is possible to use mixture fraction (or mass fraction of $N_{2}$ ) and one reaction progress variable (mass fraction of $\mathrm{CO}_{2}$ ), because the result of the REDIM calculations show that they are suitable coordinates. Thus, in the present work, $m=2$. Equal diff usivities and unity Lewis number are assumed in the present application of REDIM, although it is possible to extend the REDIM concept to systems with non-equal diffusivities.

In the following, we describe only the specific implementation details for the considered system. Let $\boldsymbol{\Phi}$ denote the thermo-kinetic state $\left(\boldsymbol{\Phi}=\left(h, p, Y_{1} / M_{1}, \ldots, Y_{S} / M_{S}\right)^{T}\right)$, where $h$ is the specific enthalpy, $p$ the pressure, $Y$ the mass fractions, $M$ the molar masses, and $S$ the number of species. For the two-dimensional REDIM, this state vector is assumed to be a function of the two mentioned reduced coordinates. As initial guess for the REDIM, we build up a tensor product mesh covering the entire domain from the un-burnt mixing line to the line of complete reaction to $\mathrm{H}_{2} \mathrm{O}$ and $\mathrm{CO}_{2}$. Values inside the domain are estimated, based on an interpolation between 
Fig. 1 Initial guess for the REDIM; blue: complete reaction to $\mathrm{CO}_{2}$ and $\mathrm{H}_{2} \mathrm{O}$; magenta: unburned mixture; red and green: two laminar diffusion flamelets for tangential pressure gradient equal to $10^{4} \mathrm{~N} / \mathrm{m}^{4}$ and $10^{6} \mathrm{~N} / \mathrm{m}^{4}$; axes: mass fractions, divided by molar mass $(\mathrm{mol} / \mathrm{kg})$

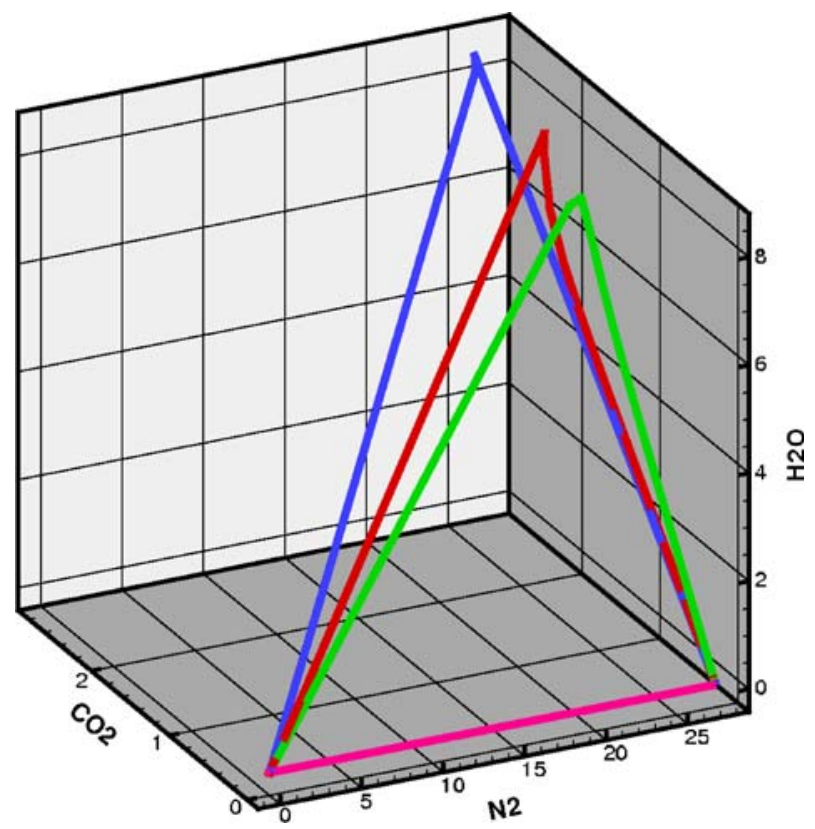

laminar flamelet solutions for varying strain rates (diffusion flamelets), see Fig. 1. Figure 2 shows a projection of the tensor product grid into the $\mathrm{N}_{2} / \mathrm{CO}_{2} / \mathrm{H}_{2} \mathrm{O}$ space. Together with estimates for the initial values, estimates for the gradients of the

Fig. 2 Example of the tensor product mesh, plotted in $\mathrm{N}_{2} / \mathrm{CO}_{2} / \mathrm{H}_{2} \mathrm{O}$ space; axes: mass fractions, divided by molar mass $(\mathrm{mol} / \mathrm{kg})$

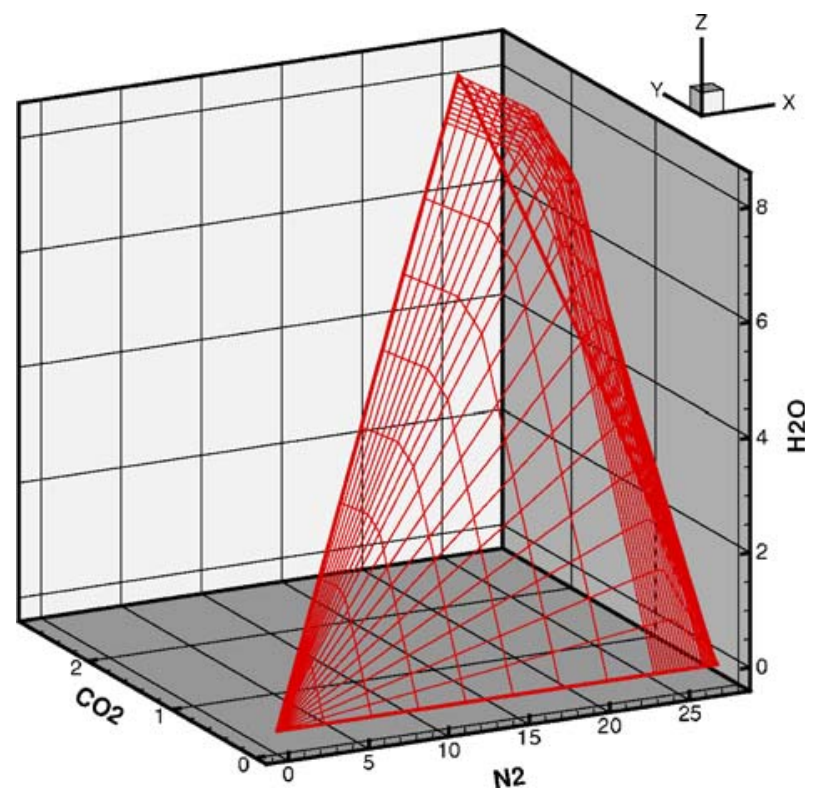


progress variables are obtained by a linear interpolation (gradients are also tabulated in terms of the tensor product mesh).

In this way we obtain $\boldsymbol{\Phi}^{0}=\boldsymbol{\Phi}^{0}(\boldsymbol{\phi})$, where $\boldsymbol{\phi}$ is the two-dimensional vector of reduced coordinates. This starting solution then evolves according to the REDIM equation:

$$
\frac{\partial \boldsymbol{\Phi}}{\partial \tau}=\left(\boldsymbol{I}-\boldsymbol{\Phi}_{\boldsymbol{\phi}} \boldsymbol{\Phi}_{\boldsymbol{\phi}}^{+}\right) \cdot\left\{\boldsymbol{F}(\boldsymbol{\Phi})+\sum_{i j} \frac{d}{\rho} \varpi_{i} \boldsymbol{\Phi}_{\phi_{i} \phi_{j}} \varpi_{j}\right\}
$$

Here $\boldsymbol{\Phi}_{\phi}$ denotes the matrix of partial derivatives of $\boldsymbol{\Phi}$ with respect to $\boldsymbol{\phi}$ (i.e., the tangent space of the manifold), $\boldsymbol{F}(\boldsymbol{\Phi})$ the vector of chemical source terms, $\rho$ the density, $d$ the diffusion coefficient, $\boldsymbol{\Phi}_{\phi_{i} \phi_{j}}$ the second partial derivatives of $\boldsymbol{\Phi}$ with respect to $\phi_{i}$ and $\phi_{j}$, and $\varpi_{i}$ denotes estimates for the spatial gradients of the reduced coordinates. As discussed in [15], it is necessary to estimate the magnitude of the spatial gradients of the reduced coordinates, but the REDIM does (in many cases) not depend very much on the choice of this estimate. In this work, we estimate the gradients based on typical results from the one-dimensional laminar flamelet calculations, which have been used to create the initial guess (see above). In fact, even for premixed flames, the REDIM, calculated based on gradient estimates from diffusion flames, produces reasonable results (see Fig. 3), which is important to capture re-ignition processes in flames with local extinction. The evolution equation is solved using a simple semi-implicit procedure. The result is a two-dimensional manifold in the state space in terms of the reduced coordinates. This manifold, as

Fig. 3 Two-dimensional REDIM, plotted in $\mathrm{N}_{2} / \mathrm{CO}_{2} / \mathrm{OH}$ space; axes: mass fractions divided by molar mass ( $\mathrm{mol} / \mathrm{kg})$, green curve: trajectory of a premixed flat flame, blue curve: trajectory of a diffusion flame

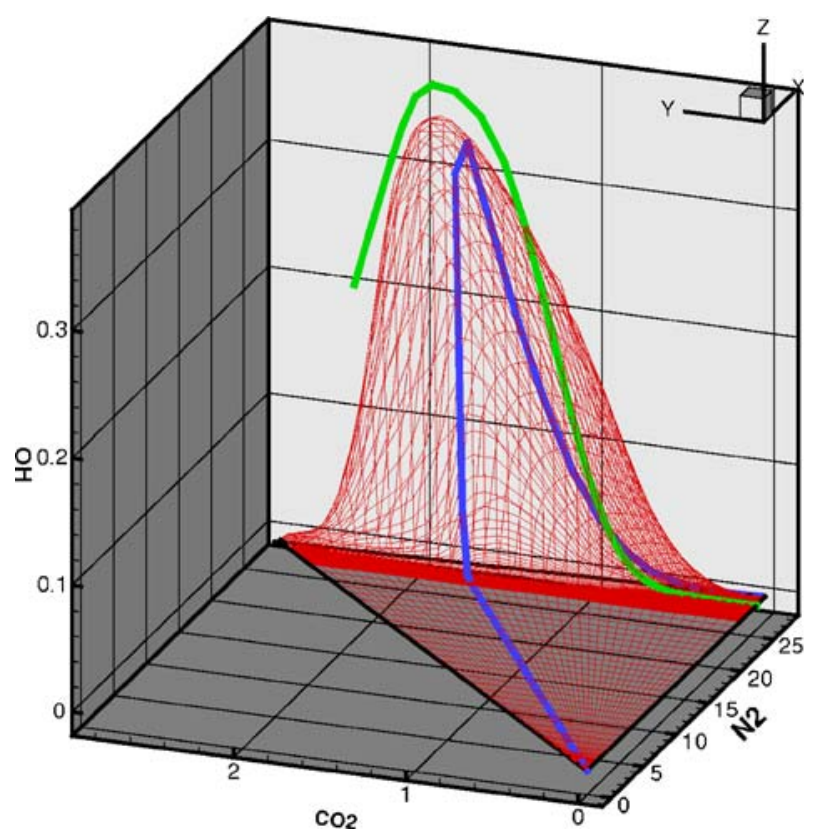



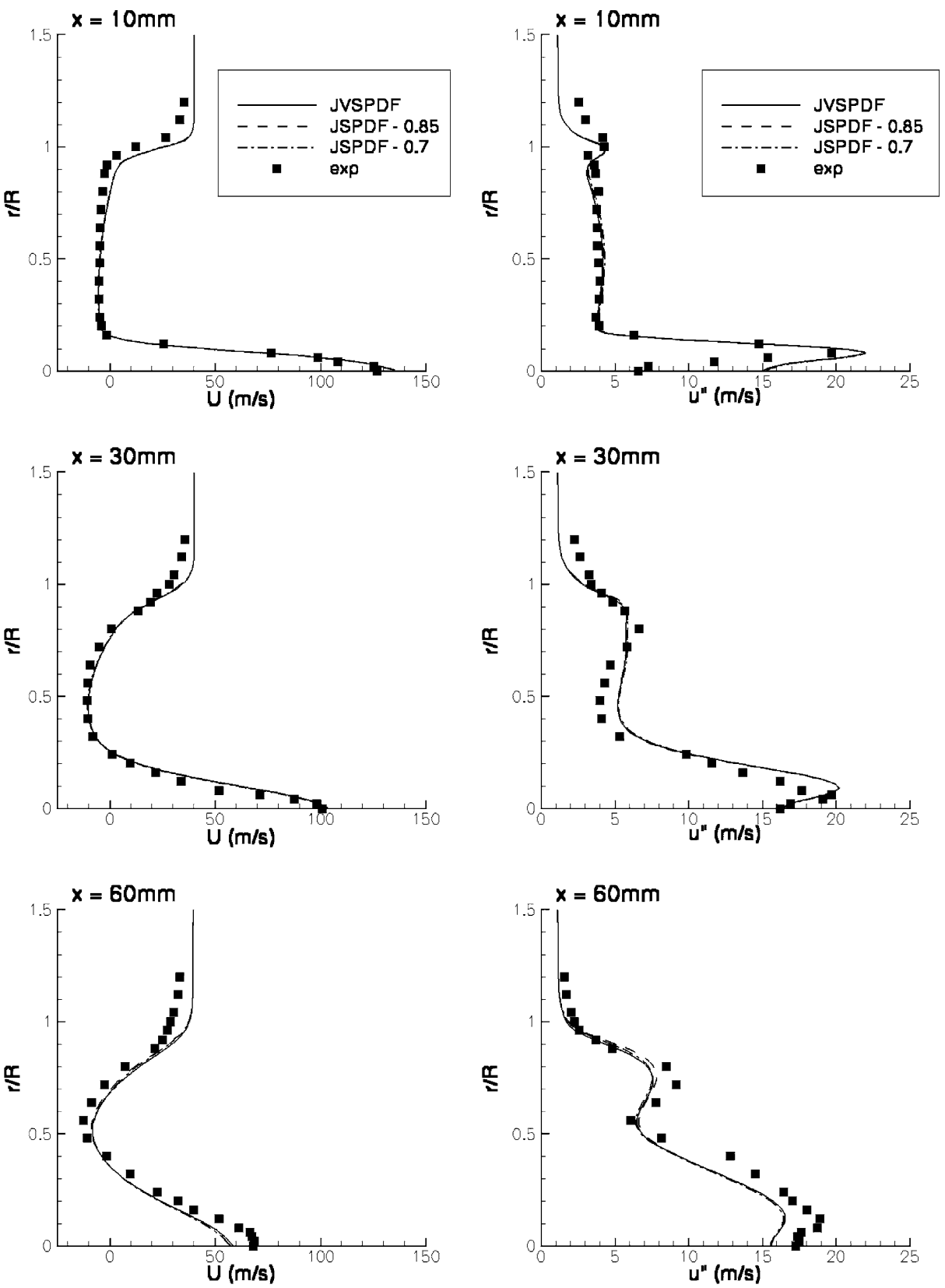

Fig. 4 Mean axial velocity component (left) and axial velocity fluctuations (right) for HM1

well as additional information such as chemical rates of the progress variable and temperature, is tabulated using the tensor product mesh.

In Eqs. 4 and 6, the composition vector is thus reduced to $\phi=\left(\xi, Y_{\mathrm{CO} 2}\right)$ and the chemical source term $S_{\mathrm{CO} 2}(\phi)$ is given by the REDIM reduced chemistry. Note that 

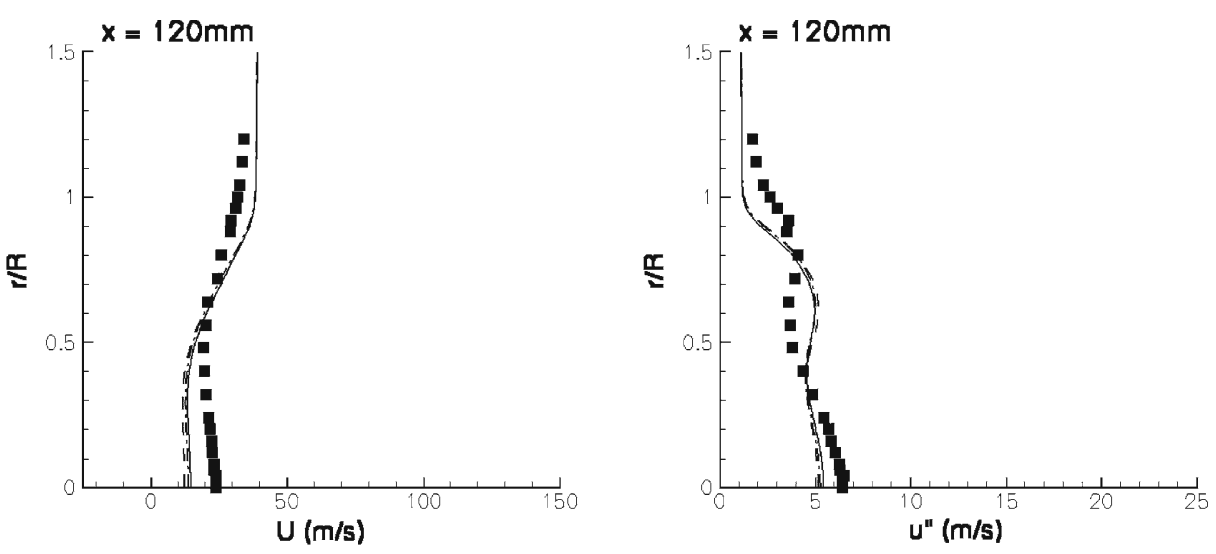

Fig. 4 Continued

in the experimental data below, the mixture fraction $\xi$ is defined on the basis of Bilger's formula [30]:

$$
\xi=\frac{\frac{2\left(Z_{C}-Z_{C, o}\right)}{W_{C}}+\frac{\left(Z_{H}-Z_{H, o}\right)}{2 W_{H}}-\frac{Z_{O}-Z_{O, o}}{W_{O}}}{\frac{2\left(Z_{C, f}-Z_{C, o}\right)}{W_{C}}+\frac{\left(Z_{H, f}-Z_{H, o}\right)}{2 W_{H}}-\frac{Z_{O, f}-Z_{O, o}}{W_{O}}}
$$

where $\mathrm{Z}_{\beta}$ is the total mass fraction of element $\beta$ and $\mathrm{W}_{\beta}$ is its atomic mass. The subscripts "f" and "o" refer to the fuel and oxidant streams. In the present numerical results, mixture fraction is defined as:

$$
\xi=1-\frac{Y_{N_{2}}}{Y_{N_{2}, o}}
$$

Differences between expressions (15) and (16) are possible due to differential diffusion effects. These are assumed small for the test case under study. Note that mixture fraction was not required for the construction of the tables with REDIM. Thus, possible differences in mixture fraction will only appear in the post-processing analysis.

\subsection{Micro-mixing model}

As micro-mixing model, the modified Curl's CD model [21] is used, with $C_{\phi}=2.0$, as in [4]. This micro-mixing model prescribes the evolution of particle composition as a series of pair-wise mixing events. The participating mixing particles are chosen at random from the set of particles present in a finite volume cell and their compositions change in the direction of the interaction partner. The degree of mixing in a pair is determined by a random variable, uniformly distributed between 0 (no mixing) and 1 (complete mixing). 

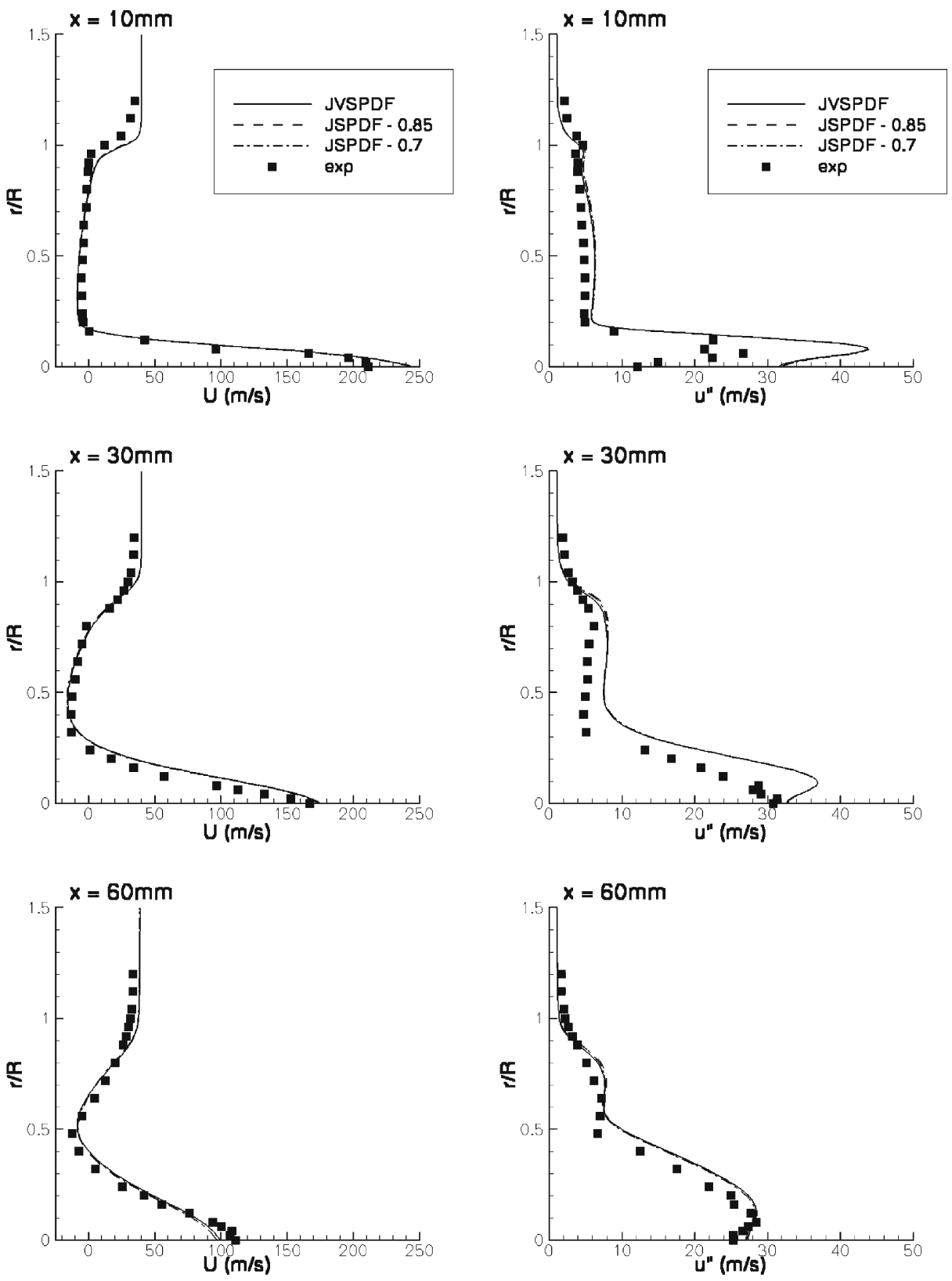

Fig. 5 Mean axial velocity component (left) and axial velocity fluctuations (right) for HM3 

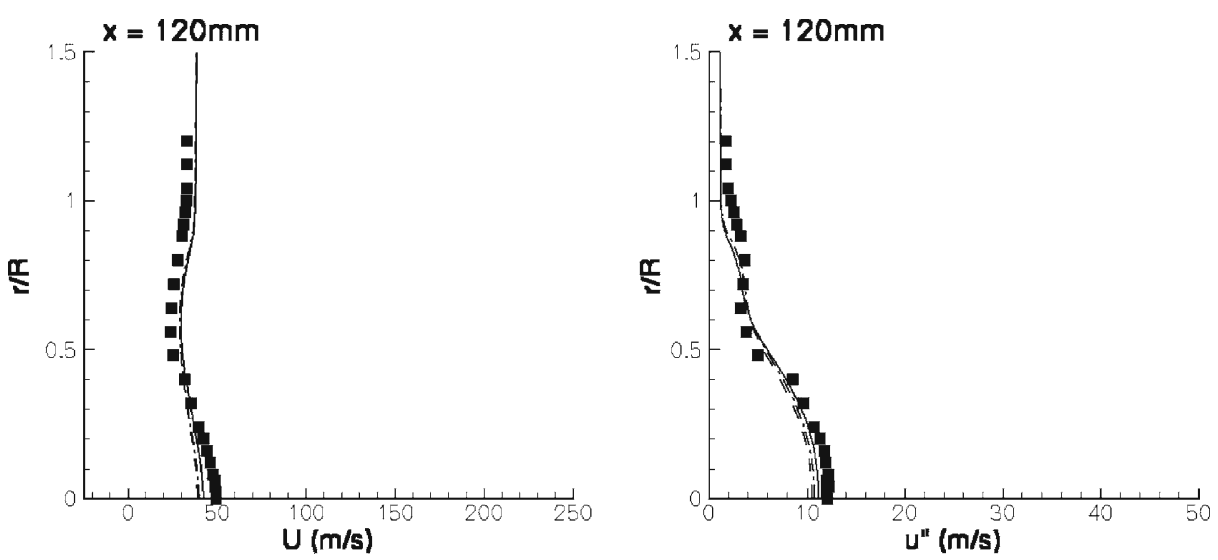

Fig. 5 Continued
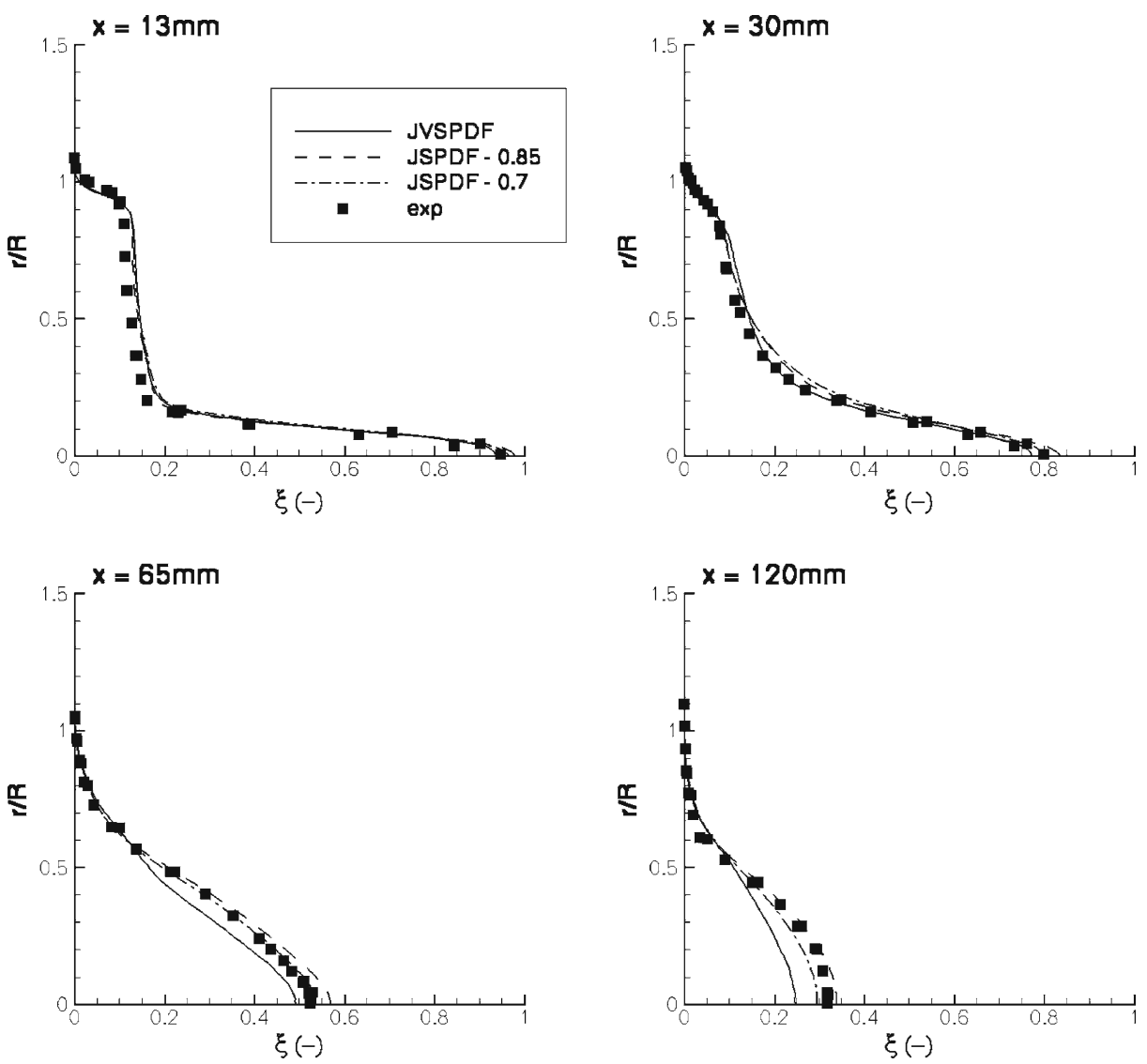

Fig. 6 Radial profiles of mean mixture fraction (HM1) 

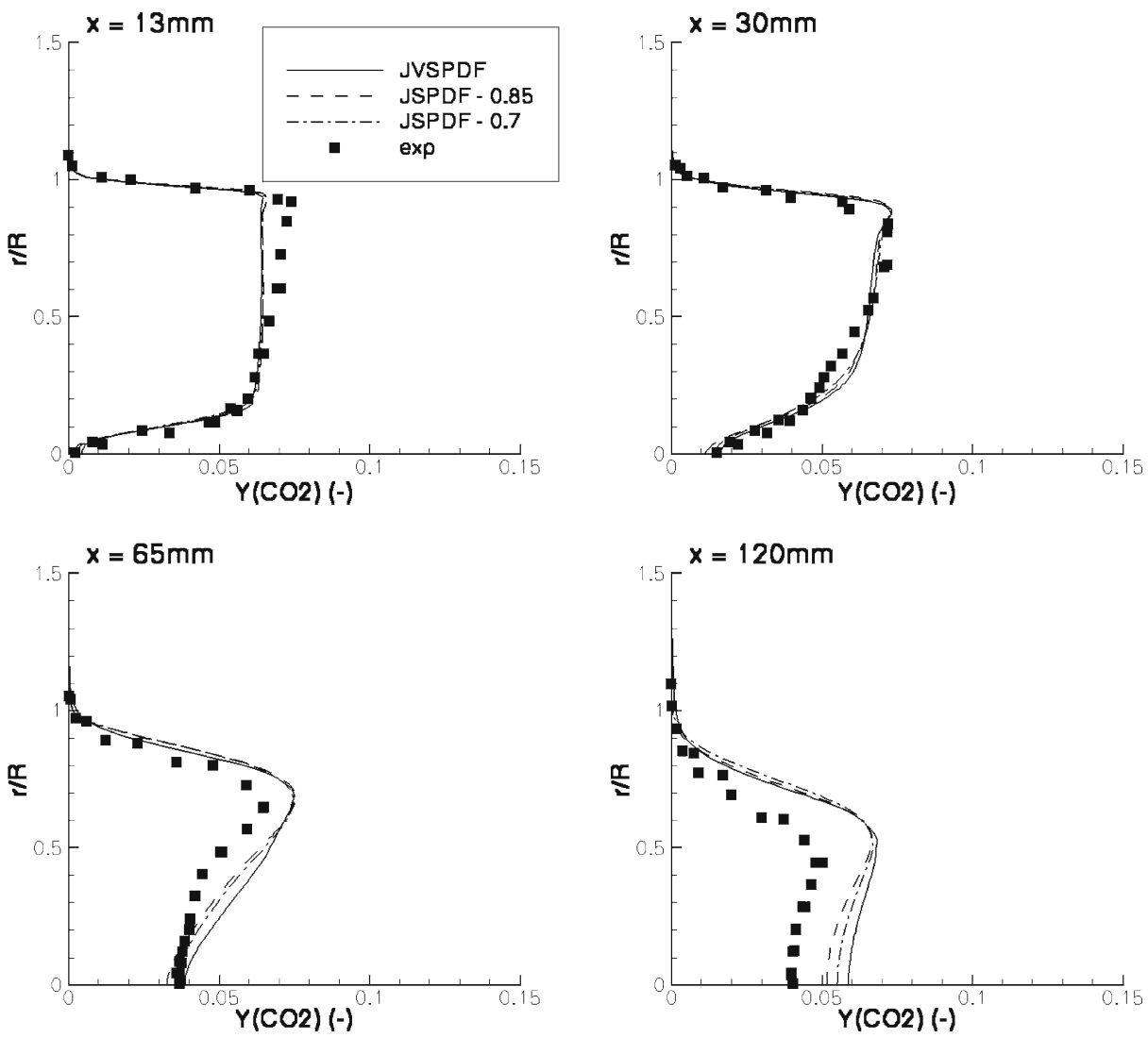

Fig. 7 Radial profiles of $\mathrm{CO}_{2}$ mass fraction (HM1)

3.4 Implications for modeled equations for mean scalar, scalar variance and scalar flux

In the simulations, Eqs. 4 and 6 are solved with a Monte Carlo method. Integration Eq. 4 or 6 over the sample space yields the same mean scalar and scalar variance transport equations:

$$
\begin{gathered}
\frac{\partial\langle\rho\rangle \widetilde{\phi}_{\alpha}}{\partial t}+\frac{\partial\langle\rho\rangle \widetilde{\phi}_{\alpha} \widetilde{U}_{j}}{\partial x_{j}}=-\frac{\partial\langle\rho\rangle \widetilde{u_{j}^{\prime \prime} \phi_{\alpha}^{\prime \prime}}}{\partial x_{j}}+\langle\rho\rangle \widetilde{S}_{\alpha}, \\
\frac{\partial\langle\rho\rangle \widetilde{\phi_{\alpha}^{\prime \prime 2}}}{\partial t}+\frac{\partial\langle\rho\rangle \widetilde{U_{j}} \widetilde{\phi_{\alpha}^{\prime \prime 2}}}{\partial x_{j}}+2\langle\rho\rangle \widetilde{u_{j}^{\prime \prime} \phi_{\alpha}^{\prime \prime}} \frac{\partial \widetilde{\phi}_{\alpha}}{\partial x_{j}}=-\frac{\partial\left\langle\rho u_{j}^{\prime \prime} \phi_{\alpha}^{\prime \prime 2}\right\rangle}{\partial x_{j}}-2\langle\rho\rangle \widetilde{\phi_{\alpha}^{\prime \prime} S_{\alpha}}-2\left\langle\rho \phi_{\alpha}^{\prime \prime} \theta_{\alpha}\right\rangle .
\end{gathered}
$$

There is no implicit summation over index $\alpha$. The observation that there is no micromixing term in Eq. 17 is a direct consequence of the conservation of the mean by the mixing model. The final term in Eq. 18, concerning the modeling of the scalar 

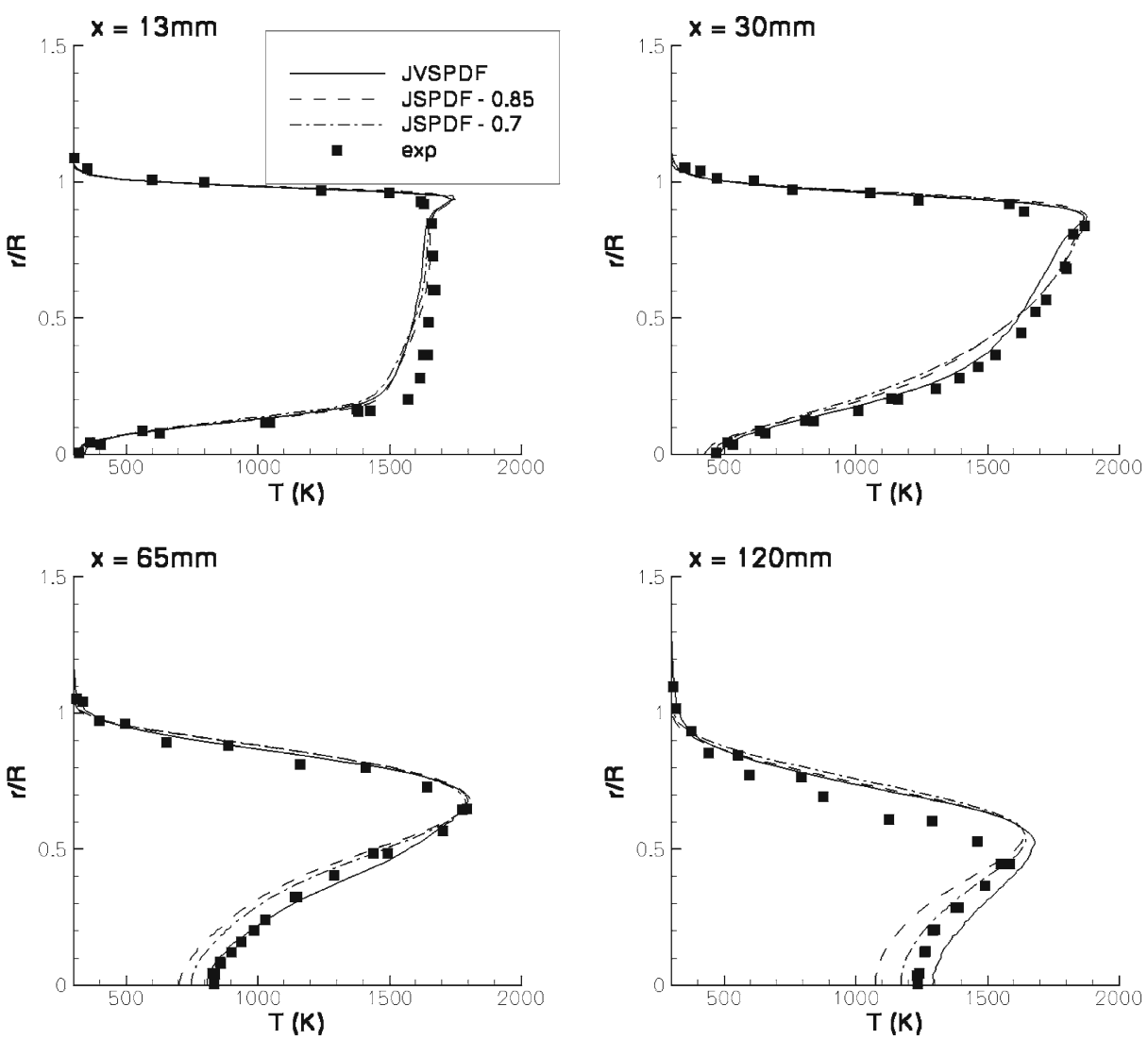

Fig. 8 Radial profiles of mean temperature (HM1)

dissipation rate and molecular diff usion, results from the mixing model. It is the same in both PDF approaches.

With the modeled joint scalar MDF, the gradient diffusion assumption, in accordance to the random walk model in the particle method, implies algebraic models for velocity-scalar correlations:

$$
\left\langle\rho u_{j}^{\prime \prime} \phi_{\alpha}^{\prime \prime}\right\rangle=-\Gamma_{T} \frac{\partial \widetilde{\phi}_{\alpha}}{\partial x_{j}} \text { and }\left\langle\rho u_{j}^{\prime \prime} \phi_{\alpha}^{\prime \prime 2}\right\rangle=-\Gamma_{T} \frac{\partial \widetilde{\phi_{\alpha}^{\prime \prime 2}}}{\partial x_{j}} .
$$

When the joint velocity-scalar MDF is considered, a differential scalar flux model is implied, depending on the Langevin model $a_{i}$ and the mixing model $\theta_{\alpha}$. For instance, the modeled scalar flux transport equation takes the form:

$$
\begin{array}{r}
\frac{\partial\langle\rho\rangle \widetilde{u_{i}^{\prime \prime} \phi_{\alpha}^{\prime \prime}}}{\partial t}+\frac{\partial\langle\rho\rangle \widetilde{U_{j}} \widetilde{u_{i}^{\prime \prime} \phi_{\alpha}^{\prime \prime}}}{\partial x_{j}}+\langle\rho\rangle \widetilde{u_{j}^{\prime \prime} \phi_{\alpha}^{\prime \prime}} \frac{\partial \widetilde{U}_{i}}{\partial x_{j}}+\langle\rho\rangle \widetilde{u_{i}^{\prime \prime} \phi_{j}^{\prime \prime}} \frac{\partial \widetilde{\phi}_{\alpha}}{\partial x_{j}} \\
=-\frac{\partial\left\langle\rho u_{j}^{\prime \prime} u_{i}^{\prime \prime} \phi_{\alpha}^{\prime \prime}\right\rangle}{\partial x_{j}}+\langle\rho\rangle \widetilde{u_{i}^{\prime \prime} S_{\alpha}}+\left\langle\rho a_{i} \phi_{\alpha}^{\prime \prime}\right\rangle+\left\langle\rho u_{i}^{\prime \prime} \theta_{\alpha}\right\rangle .
\end{array}
$$



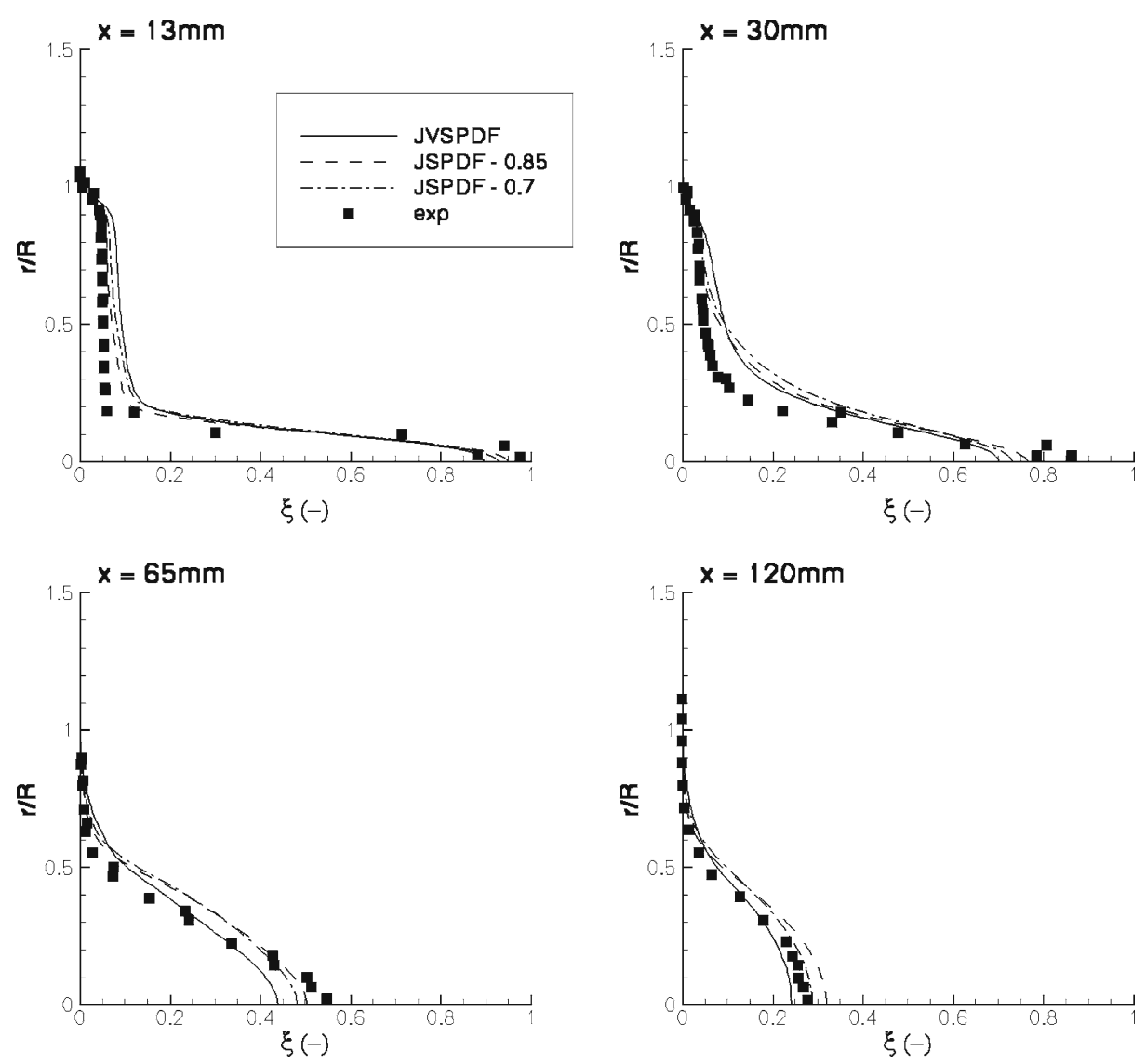

Fig. 9 Radial profiles of mean mixture fraction (HM3)

Thus, there is a difference in modeling of the scalar flux and higher order velocityscalar fluctuation correlations.

Note that Eqs. 17, 18 and 19 or 20, are solved as a result of the Monte Carlo method, applied to Eq. 4 or 6 , in the sense that the moments of the computed JSPDF or JVSPDF satisfy Eqs. 17, 18 and 19 or 20.

\section{Test Case}

The fuel (50\% $\mathrm{H}_{2}$ and $50 \% \mathrm{CH}_{4}$ by volume) is injected in the centre of the bluff-body burner through an injector of diameter $D_{j}=3.6 \mathrm{~mm}$. The bluff body, of diameter $D_{b}=50 \mathrm{~mm}$, is surrounded by an unconfined co-flowing air stream. Fuel and air mix in the recirculation zone behind the bluff body, where chemical reactions occur. The resulting hot products stabilize the flame.

In HM1, the jet and co-flow bulk velocities are respectively $118 \mathrm{~m} / \mathrm{s}$ and $40 \mathrm{~m} / \mathrm{s}$, while for HM3, the fuel jet velocity is $214 \mathrm{~m} / \mathrm{s}$ [5, 6]. The numerical settings are described in [23]. A $6 D_{b}$ long and $3 D_{b}$ wide 2D computational domain is used. 

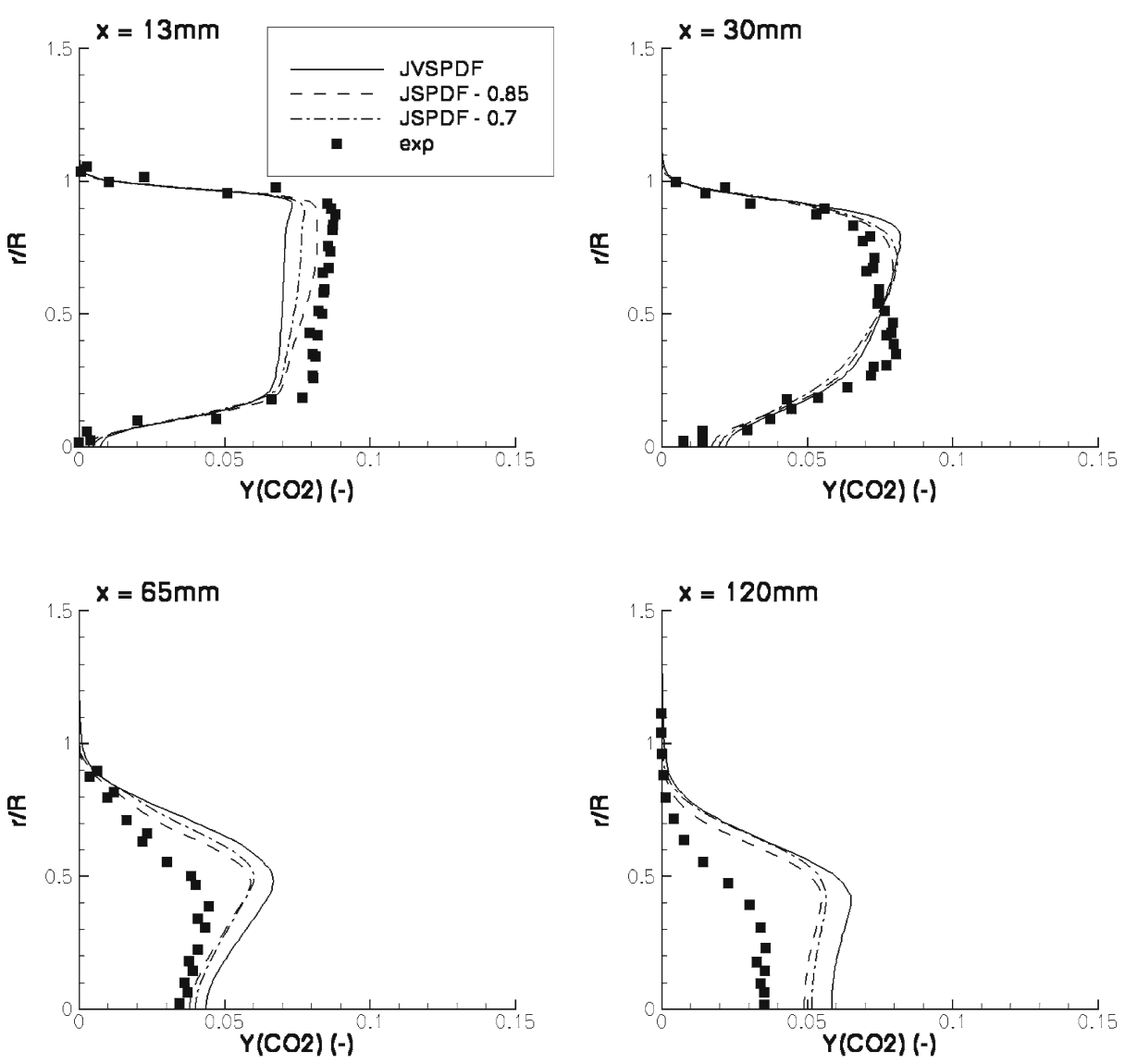

Fig. 10 Radial profiles of $\mathrm{CO}_{2}$ mass fraction (HM3)

Free-slip boundary conditions are prescribed on the bluff-body surface and on the lateral computational domain boundary. A convective outlet boundary condition, as presented in [30], is used in order to avoid reflecting waves. Inlet boundary conditions are specified at cell centers in the same way as in [23]. The simulations are performed on a $160 \times 128$ Cartesian grid, with stretching in axial and radial directions. An average of 100 particles per cell is used. Iteration averages are made over 500 iterations. Converged results, obtained with an assumed-shape PDF method, are used as initial conditions. About 1,000 outer iterations (5,000 particle time steps) are sufficient to reach a stationary solution. Results obtained after 15,000 particle time steps are now discussed.

\section{Results and Discussion}

The focus of the present study is on the comparison between the JSPDF and the JVSPDF approach. Therefore, we restrict ourselves to presenting only the results 

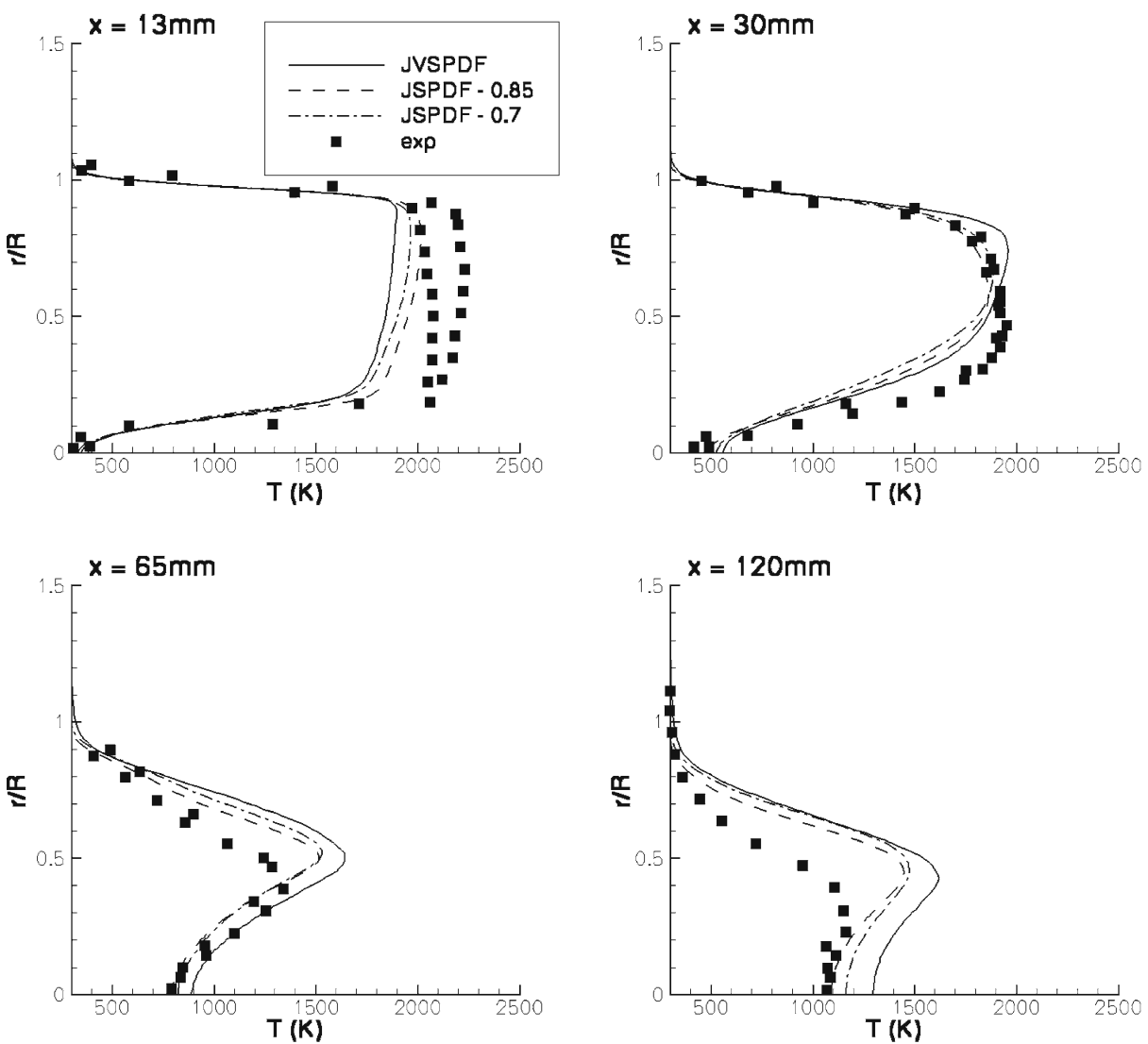

Fig. 11 Radial profiles of mean temperature (HM3)

that are relevant to this study and do not present any results for minor species mass fractions. For the JSPDF results, we focus on the default value $S c_{T}=0.7$. The results with $S c_{T}=0.85$ are added to illustrate the effect of turbulent diffusion.

\subsection{Mean velocity and Reynolds stresses}

Figures 4 and 5 show the results for mean axial velocity and axial velocity fluctuations. They are very similar to the results of [10,23], or [26]. This is also true for the mean radial velocity component and the other Reynolds stresses (not shown). Good agreement with experimental data is found within the recirculation zone $(\mathrm{x}<50 \mathrm{~mm})$. The agreement deteriorates in the neck zone $(50 \mathrm{~mm}<x<90 \mathrm{~mm})$ and the jet-like zone $(\mathrm{x}>90 \mathrm{~mm})$. These downstream discrepancies have been observed in the different RANS calculations presented at the TNF workshops [7].

An important observation for the present study is that the very small differences between scalar PDF and velocity-scalar PDF results (due to some small differences in mean density) are negligible, even for flame HM3. 

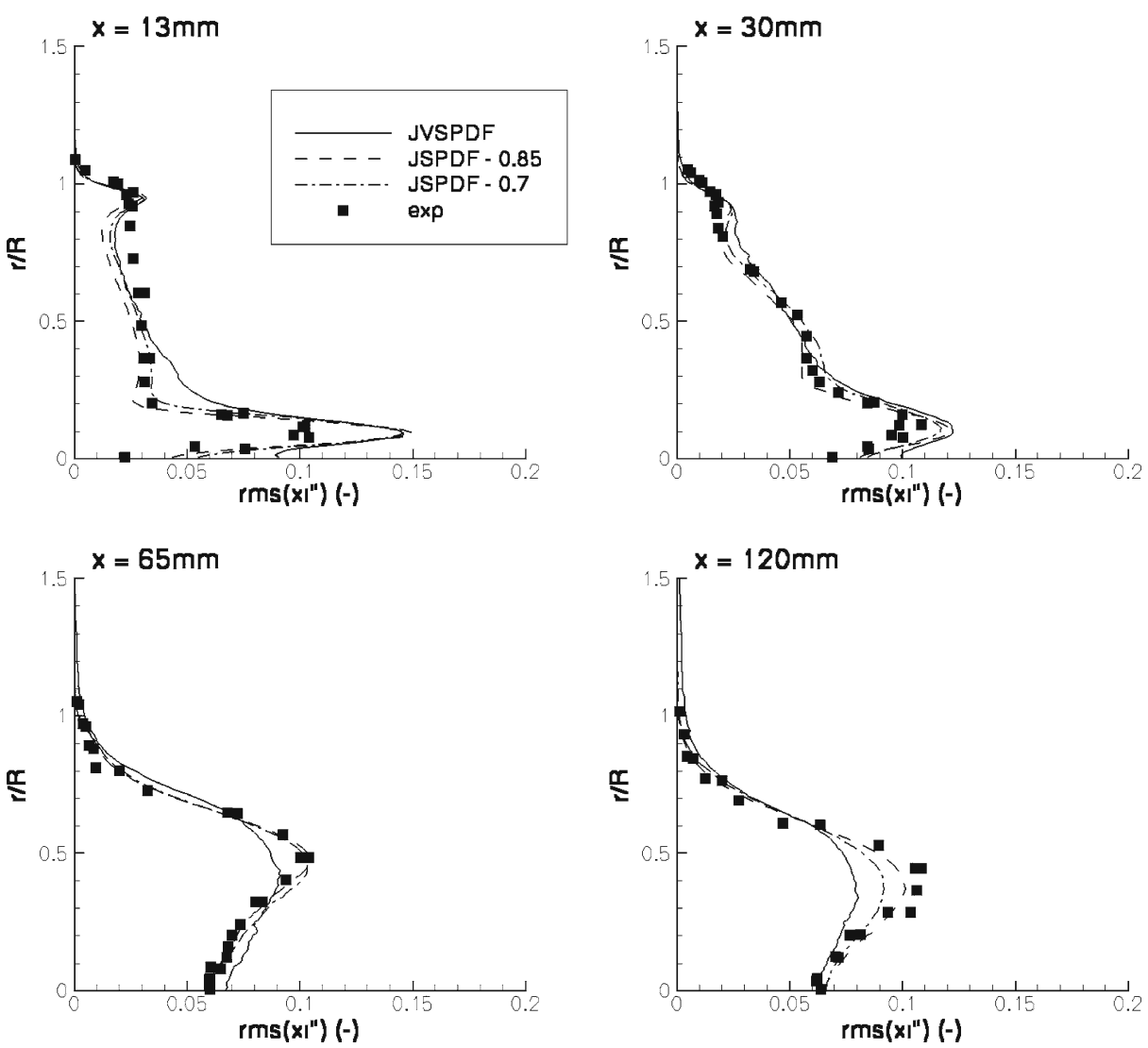

Fig. 12 Radial profiles of mixture fraction variance (HM1)

\subsection{Mean composition}

Figures 6, 7, 8 show radial profiles for mean mixture fraction, $\mathrm{CO}_{2}$ mass fraction and temperature for flame HM1. Significant differences between JSPDF and JVSPDF results are observed. Within the recirculation region, where good agreement is obtained for the mean velocity and turbulent velocity fluctuations, the quality of the JVSPDF mean mixture fraction results is better than the JSPDF results. Indeed, excessive radial turbulent diffusion is observed with JSPDF in this region, where radial gradients of mean velocity and mixture fraction are large. Differences in $\mathrm{CO}_{2}$ mass fractions are small in this region. The differences in mean mixture fraction predictions are reflected in better mean temperature predictions with JVSPDF than with JSPDF. In the neck zone, mean mixture fraction is in general under-predicted with the JVSPDF approach, though. This is in line with the under-prediction of mean axial velocity in this region. In the JSPDF results, agreement with experimental data seems better, despite the under-prediction of mean axial velocity (and thus convection). As the convective terms in the modeled transport equations are very similar and the turbulence level is practically the same, too, the differences between 

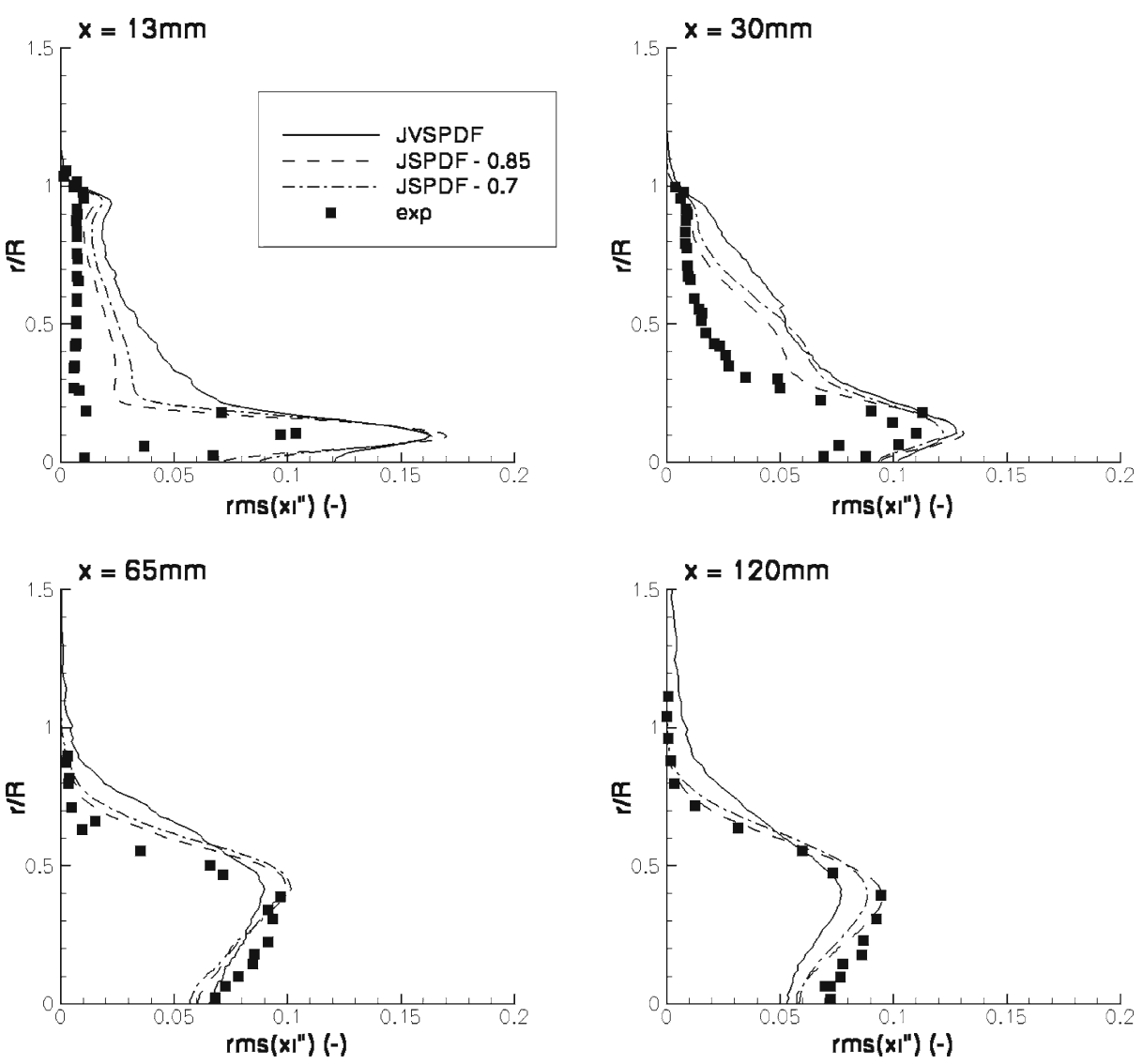

Fig. 13 Radial profiles of mixture fraction variance (HM3)

JVSPDF and JSPDF must be due to differences in the scalar fluxes. Thus, there seems to be too little turbulent diffusion in the JSPDF results, compensating for the underprediction of mean turbulent convection. With JSPDF, it is clearly possible to affect the results through this parameter. It is recalled here that, with JVSPDF, there is no 'degree of freedom' of playing with the turbulent Schmidt number to improve the results.

We also note that the mean temperatures are in general somewhat lower in the JSPDF simulations. As the level of local extinction is comparable to the JVSPDF results (see the section on scatter plots below), the mean temperature differences are primarily due to differences in mean mixture fraction. This is in line with the observation in Fig. 5 that the mean $\mathrm{CO}_{2}$ mass fraction value, the progress variable in REDIM in this study, is also lower in this region in the JSPDF results, which is logical as the mean mixture fraction value is further away from stoichiometric, on the rich side, than in the JVSPDF results.

Figures 9, 10, 11 reveal that the observations are very similar for the computational results of flame HM3. The temperatures obtained for HM3 are somewhat lower 

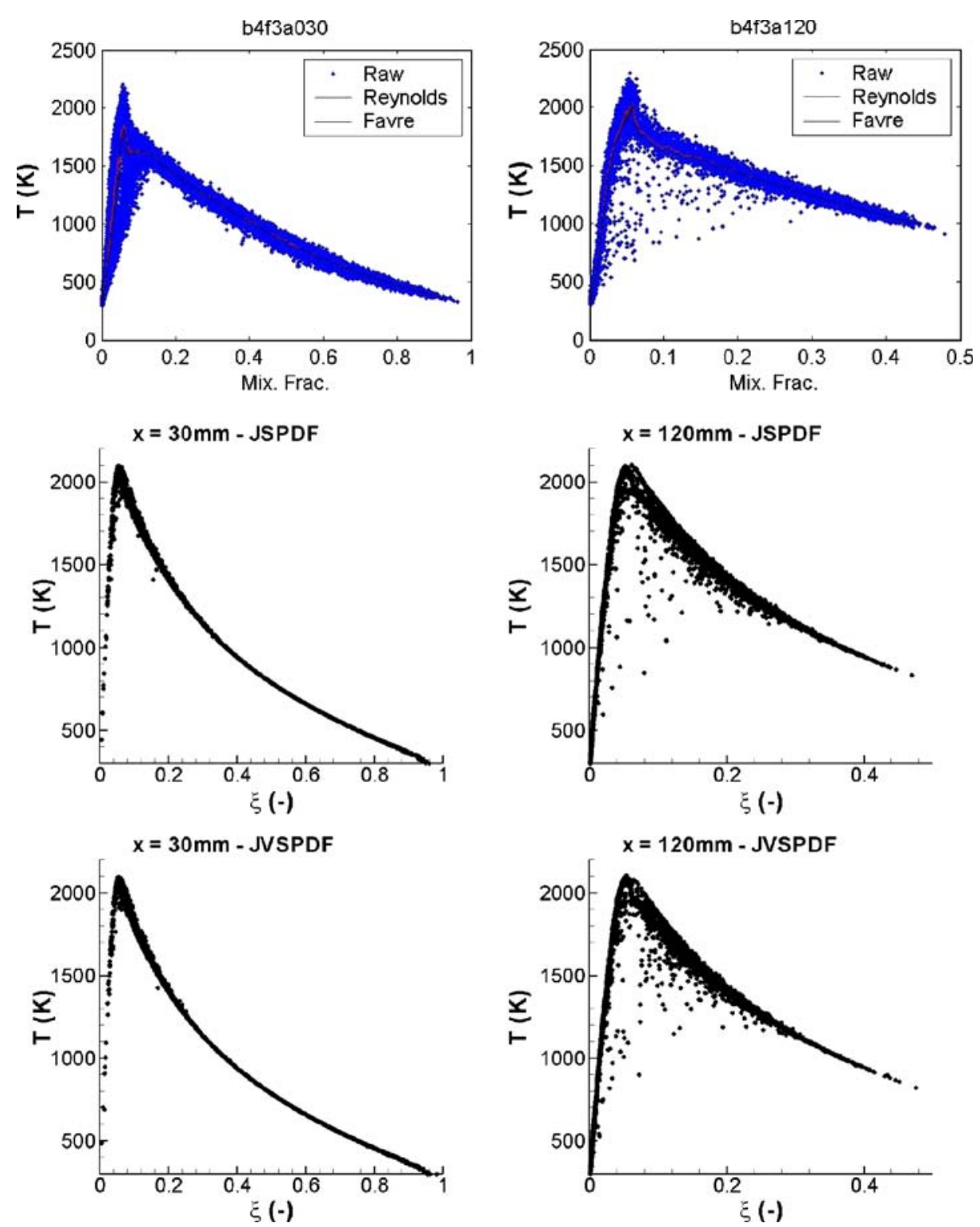

Fig. 14 Scatter plots for temperature (HM1). Experimental plots [7] reveal the 'raw' data (scatter plots) as well as the conditional 'Reynolds' and 'Favre' averages (not discussed here). Results for JSPDF are with $S c_{T}=0.7$. Results with $S c_{T}=0.85$ are almost identical

than for HM1, due to the occurrence of more local extinction (see below). There is no excellent agreement with experimental data, though: neither the JVSPDF results nor the JSPDF results capture the experimental observation that the reaction region shifts from the outer side of the recirculation region (as in HM1) to the inner side. Note that, in [4], it is reported that, in contrast to experimental observations, 

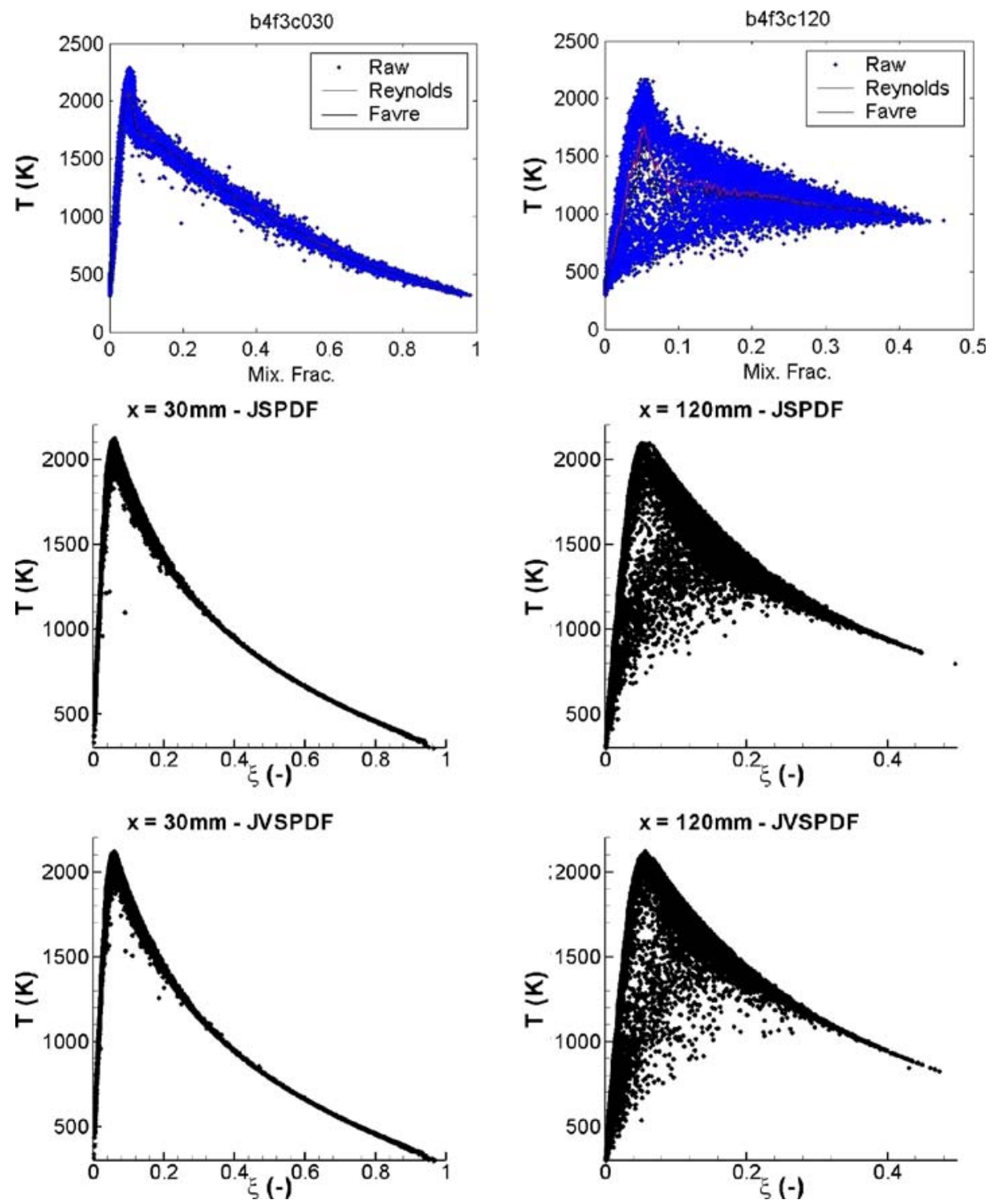

Fig. 15 Scatter plots for temperature (HM3). Experimental plots [7] reveal the 'raw' data (scatter plots) as well as the conditional 'Reynolds' and 'Favre' averages (not discussed here). Results for JSPDF are with $S c_{T}=0.7$. Results with $S c_{T}=0.85$ are almost identical

no stationary solution could be obtained for JSPDF results with the CD mixing model for flame HM3. In the present study, the limit cycle, reported in [4], is not found, probably due to the application of the REDIM chemistry model instead of a $C_{1}$ skeletal mechanism. Better agreement with experimental data can also most probably be obtained by applying more detailed chemistry mechanisms. 


\subsection{Mixture fraction variance}

In Figs. 12 and 13, differences are observed in the radial profiles of mixture fraction variance. In general, the JSPDF results are in better correspondence with the experimental data. The results are slightly better than reported in [4]. It is clear that the observed differences between JVSPDF and JSPDF are related to differences in mean mixture fraction, as well as to the modeling of velocity-scalar correlations: the scalar flux $\widetilde{u_{j}^{\prime \prime} \xi^{\prime \prime}}$, appearing in the production term, and the triple correlation $\widetilde{u_{j}^{\prime \prime} \xi^{\prime \prime 2}}$, appearing in the turbulent diffusion term in Eq. 18. The under-prediction of mixture fraction variance with JVSPDF in the neck zone is mainly due to the lower mean mixture fraction values in this region.

\subsection{Results in composition space: scatter plots}

Figures 14 and 15 reveal that, in composition space, differences between JSPDF and JVSPDF results are small, with the turbulence, chemistry and micro-mixing models applied. There is slightly more local extinction with JSPDF.

This indicates that the effect of differences in particle evolution in physical space due to mean convection and turbulent mixing, is small in composition space. Consequently, after further evolution due to the chemical source term, as retrieved from the REDIM table as constructed for the chemistry mechanism under consideration, the global scatter plots are very similar for JSPDF and JVSPDF simulations.

\section{Conclusions}

A comparison of scalar PDF and velocity-scalar PDF modeling has been conducted for the bluff-body flames HM1 and HM3. Differences in the predicted turbulent flow fields are negligible. We applied one turbulence (LRR-IPM), chemistry (REDIM) and micro-mixing (CD) model.

Significant differences are observed in results for mean mixture fraction (and mean $\mathrm{CO}_{2}$ mass fraction). Consequently, there are also strong deviations in mean temperature and mixture fraction variance. These are attributed to implied modeling differences in the scalar fluxes (and higher order correlations). In general, joint velocity-scalar PDF results, potentially closer to physical reality due to a differential scalar flux model, are in line with the turbulent flow field results. In the recirculation region, with strong radial gradients of mean velocity and mean mixture fraction, the JVSPDF results are somewhat better, as excessive turbulent diffusion occurs in the JSPDF simulations, in which a gradient diffusion assumption is implied. In the neck zone, however, the JVSPDF results are not better than joint scalar PDF results, due to poorer agreement to experimental data for the turbulent flow field. Local tuning of the turbulent Schmidt number in JSPDF simulations, which is impossible in JVSPDF simulations, can compensate for this for the test case under study.

The impact of the choice, JSPDF vs. JVSPDF, on scatter plots is small for the test cases under study, with a slightly higher level of local extinction in the JSPDF results. The scatter plots are in general in good agreement with experimental data. 
Acknowledgements This collaborative research is supported by the COMLIMANS program, by the Spanish MEC under Project ENE 2005-09190-C04-04/CON, by the Deutsche Forschungsgemeinschaft and by the Fund of Scientific Research-Flanders (Belgium) (FWO-Vlaanderen) through FWO-project G.0079.07.

\section{References}

1. Pope, S.B.: PDF methods for turbulent reactive flows. Prog. En. Combust. Sci. 11, 119-192 (1985)

2. Ren, Z., Pope, S.B.: An investigation of the performance of turbulent mixing models. Combust. Flame. 136, 208-216 (2004)

3. Merci, B., Roekaerts, D., Naud, B.: Study of the performance of three micromixing models in transported scalar PDF simulations of a piloted jet diffusion flame ("Delft Flame III"). Combust. Flame. 144, 476-493 (2006)

4. Merci, B., Roekaerts, D., Naud, B., Pope, S.B.: Comparative study of micromixing models in transported scalar PDF simulations of turbulent nonpremixed bluff body flames. Combust. Flame. 146, 109-130 (2006)

5. Dally, B.B., Masri, A.R.: Flow and mixing fields of turbulent bluff-body jets and flames. Combust. Theory Mod. 2, 193-219 (1998)

6. Dally, B.B., Masri, A.R., Barlow, R.S., Fiechtner, G.J.: Instantaneous and mean compositional structure of bluff-body stabilized nonpremixed flames. Combust. Flame. 114, 119-148 (1998)

7. http://www.aeromech.usyd.edu.au/thermofluids/main_frame.htm

8. Cao, R.R., Pope, S.B.: The influence of chemical mechanisms on PDF calculations of nonpremixed piloted jet flames. Combust. Flame. 143, 450-470 (2005)

9. Merci, B., Naud, B., Roekaerts, D.: Interaction between chemistry and micro-mixing modeling in transported pdf simulations of turbulent non-premixed flames. Combust. Sci. Technol. 179, 153-172 (2007)

10. Liu, K., Pope, S.B., Caughey, D.A.: Calculations of bluff-body stabilized flames using a joint probability density function model with detailed chemistry. Combust. Flame. 141, 89-117 (2005)

11. Gkagkas, K., Lindstedt, R.P., Kuan, T.S.: Proceedings 2nd ECCOMAS thematic conference on computational combustion. In: Roekaerts, D., Coelho, P., Boersma, B.J., Claramunt, K. (eds.) Delft, 2007, pp. 16 (2007). [ISBN: 978-90-811768-1-1]

12. Lindstedt, R.P., Ozarovsky, H.C., Barlow, R.S., Karpetis, A.N.: Progression of localized extinction in high Reynolds number turbulent jet flames. Proc. Combust. Inst. 31, 1551-1558 (2007)

13. Barlow, R.S.: International workshop on measurement and computation of turbulent nonpremixed flames. http://www.ca.sandia.gov/TNF

14. Sreedhara, S., Huh, K.Y.: Modeling of turbulent, two-dimensional nonpremixed CH4/H-2 flame over a bluffbody using first- and second-order elliptic conditional moment closures. Combust. Flame. 143, 119-134 (2005)

15. Bykov, V., Maas, U.: The extension of the ILDM concept to reaction-diffusion manifolds. Combust. Theory Mod. 11(6), 839-862 (2007)

16. Warnatz, J., Maas U., Dibble, R.W.: Combustion. Springer (1996) [ISBN:3-540-60730-7]

17. Nafe, J., Maas, U.: A general algorithm for improving ILDMs. Combust. Theory Mod. 6, 697-709 (2002)

18. Ren, Z., Pope, S.B.: The use of slow manifolds in reactive flows. Combust. Flame. 147, 243-261 (2006)

19. Ren, Z., Pope, S.B.: Transport-chemistry coupling in the reduced description of reactive flows. Combust. Theory Mod. 11(5), 715-739 (2007)

20. Li, G., Naud, B., Roekaerts, D.: Numerical investigation of a bluff-body stabilised nonpremixed flame with differential Reynolds-stress models. Flow, Turbul. Combust. 70, 211-240 (2003)

21. Janicka, J., Kolbe, W., Kollmann, W.: Closure of the transport-equation for the probability density-function of turbulent scalar fields. J. Non-Equil. Thermod. 4, 47-66 (1979)

22. Subramaniam, S., Pope, S.B.: A mixing model for turbulent reactive flows based on Euclidean minimum spanning trees. Combust. Flame. 115, 487-514 (1998)

23. Naud, B., Jiménez, C., Roekaerts, D.: A consistent hybrid PDF method: implementation details and application to the simulation of a bluff-body stabilised flame. Prog. Comp.l Fluid Dyn. 6, 146-157 (2006) 
24. Muradoglu, M., Pope, S.B., Caughey, D.A.: The hybrid method for the PDF equations of turbulent reactive flows: consistency conditions and correction algorithms. J. Comp. Phys. 172, 841-878 (2001)

25. Jenny, P., Pope, S.B., Muradoglu, M., Caughey, D.A.: A hybrid algorithm for the joint PDF equation of turbulent reactive flows. J. Comp. Phys. 166, 218-252 (2001)

26. Muradoglu, M., Pope, S.B.: Local time-stepping algorithm for solving probability density function turbulence model equations. AIAA J. 40, 1755-1763 (2002)

27. Launder, B.E., Reece, G.J., Rodi, W.: Progress in development of a reynolds-stress turbulence closure. J. Fluid Mech. 68, 537-566 (1975)

28. Wouters, H.A., Peeters, T.W.J., Roekaerts, D.: On the existence of a generalized Langevin model representation for second-moment closures. Phys. Fluids. 8, 1702-1704 (1996)

29. Pope, S.B.: On the relationship between stochastic Lagrangian models of turbulence and 2ndmoment closures. Phys. Fluids. 6, 973-985 (1994)

30. Bilger, R.W., Starner, S.H., Kee, R.J.: On reduced mechanisms for methane air combustion in nonpremixed flames. Combust. Flame. 80, 135-149 (1990) 